\title{
COVID-19 Severity and Stroke: Correlation of Imaging and Laboratory Markers
}

\author{
(D).M. Katz, (D)R.B. Libman, (D).J. Wang, (D)C.G. Filippi, (D). Sanelli, (D)A. Zlochower, (D) M. Gribko, (D) S.V. Pacia, (D) R.I. Kuzniecky,
} (D) S. Najjar, and IDS. Azhar

\begin{abstract}
BACKGROUND AND PURPOSE: Coronavirus disease 2019 (COVID-19) appears to be an independent risk factor for stroke. We hypothesize that patients who develop stroke while hospitalized for severe COVID-19 will have higher inflammatory markers and distinct stroke imaging patterns compared with patients positive for COVID-19 with out-of-hospital stroke onset and milder or no COVID-19 symptoms.
\end{abstract}

MATERIALS AND METHODS: This is a retrospective case series of patients positive for COVID-19 on polymerase chain reaction testing with imaging-confirmed stroke treated within a large health care network in New York City and Long Island between March 14 and April 26, 2020. Clinical and laboratory data collected retrospectively included complete blood counts and creatinine, alanine aminotransferase, lactate dehydrogenase, C-reactive protein, ferritin, and D-dimer levels. All CT and MR imaging studies were independently reviewed by 2 neuroradiologists who recorded stroke subtype and patterns of infarction and intracranial hemorrhage.

RESULTS: Compared with patients with COVID-19 with outside-of-hospital stroke onset and milder or no COVID-19 symptoms ( $n=45,52.3 \%$ ), patients with stroke already hospitalized for severe COVID-19 ( $n=41,47.7 \%)$ had significantly more frequent infarctions $(95.1 \%$ versus $73.3 \%, P=.006)$, with multivascular distributions $(56.4 \%$ versus $33.3 \%, P=.022)$ and associated hemorrhage $(31.7 \%$ versus $4.4 \%, P=.001)$. Patients with stroke admitted with more severe COVID-19 had significantly higher C-reactive protein and ferritin levels, elevated D-dimer levels, and more frequent lymphopenia and renal and hepatic injury (all, $P<.003$ ).

CONCLUSIONS: Patients with stroke hospitalized with severe COVID-19 are characterized by higher inflammatory, coagulopathy, and tissue-damage biomarkers, supporting proposed pathogenic mechanisms of hyperinflammation activating a prothrombotic state. Cautious balancing of thrombosis and the risk of hemorrhagic transformation is warranted when considering anticoagulation.

ABBREVIATIONS: COVID-19 = coronavirus disease 2019; SARS-CoV-2 = Severe Acute Respiratory Syndrome coronavirus 2

$\mathbf{F}$ irst reported as a respiratory illness in Wuhan, China, in December 2019, ${ }^{1}$ coronavirus disease 2019 (COVID-19), caused by the novel coronavirus Severe Acute Respiratory Syndrome coronavirus 2 (SARS-CoV-2), has become a global pandemic. As of the end of August 2020, there were $>33$ million infections and $>1$ million deaths globally, including $>7$ million infections and $>206,000$ deaths in the United States alone. ${ }^{2}$ While many patients infected

Received July 29, 2020; accepted after revision October 2.

From the Departments of Neurology (J.M.K., R.B.L., M.G., S.V.P., R.I.K., S.N., S.A.), and Radiology (C.G.F., P.S., A.Z.), Donald and Barbara Zucker School of Medicine at Hofstra/Northwell, Hempstead, New York; and Feinstein Institute for Medical Research at Northwell Health (J.J.W.), Manhasset, New York.

Please address correspondence to Christopher G. Filippi, MD, Donald and Barbara Zucker School of Medicine at Hofstra/Northwell, 500 Hofstra Blvd, Hempstead, NY 11549; e-mail: cfilippi@northwell.edu; @sairaallapeikko

- Indicates open access to non-subscribers at www.ajnr.org

Indicates article with online supplemental data.

http://dx.doi.org/10.3174/ajnr.A6920 with the SARS-CoV-2 virus have mild or asymptomatic disease, ${ }^{3}$ a sizeable number of patients require hospitalization and frequently develop multiorgan dysfunction secondary to a heightened immune response. ${ }^{4,5}$ Either due to this cytokine storm or direct viral or immune-mediated endothelial injury, some patients with COVID19 develop prothrombotic and coagulopathic states, often simultaneously, and these phenomena may underlie the observed association between COVID-19 and stroke. ${ }^{5-11}$

In a previous study, we showed that COVID-19 is a strong independent risk factor for stroke in hospitalized patients. ${ }^{12}$ In this study, we compare patients positive for COVID-19 with out-ofhospital stroke onset who had mild or no COVID-19 symptoms with patients diagnosed with stroke while already hospitalized for severe COVID-19. We hypothesize that patients with stroke with severe COVID-19 will have significantly higher levels of inflammatory (C-reactive protein and ferritin) and coagulopathic markers (D-dimers) and distinctive stroke imaging patterns. Our aim is to 
provide evidence that a hyperinflammatory and prothrombotic state, such as seen in patients with severe SARS-CoV-2 infection, underlies the mechanism linking COVID-19 and stroke.

\section{MATERIALS AND METHODS}

This is a retrospective study of patients with COVID-19 concurrently diagnosed with stroke, admitted between March 14 and April 26, 2020, to 11 different Northwell Health hospitals in New York City and Long Island. Northwell Health is the largest health care network in New York, with multiple tertiary teaching hospitals and community hospitals. The institutional review board approved this Health Insurance Portability and Accountability Act-compliant study as minimal risk and waived the requirement for informed consent. Additional methods, as well as demographic, clinical, and outcome details of this cohort, have been reported elsewhere. ${ }^{12}$ Study inclusion required the following: 1) polymerase chain reaction-proved SARS-CoV-2 infection, 2) imaging-confirmed stroke, 3) documented stroke-symptom onset during a COVID-19 illness, or 4) the onset of COVID-19 symptoms or SARS-CoV-2 polymerase chain reaction positivity, within 14 days of stroke-symptom onset. The last criterion was meant to capture patients who tested positive for SARS-CoV-2 infection post-stroke hospitalization, applying the standard definition used in clinical care in terms of the potential latency between infection and symptom onset. Using this criterion, we captured 6 likely patients with initially false-negative results on polymerase chain reaction testing with a delay from COVID-19 $(n=4)$ or stroke ( $n=$ two; 2 - and 3-day delay) symptom onset to polymerase chain reaction positivity. Three additional patients who met this criterion possibly acquired COVID-19 while in the hospital, but this cannot be established with any certainty; therefore, these patients are included in our analysis. Clinical and laboratory data were collected by retrospective chart review. Only laboratory variables with at least $75 \%$ complete data were included in our analysis. Two neuroradiologists with Certificates of Added Qualification characterized the neuroimaging findings, blinded to clinical and laboratory data; in cases of disagreement, consensus was reached.

In-hospital stroke onset was defined as a new-onset focal neurologic deficit or altered mental status after hospital admission for COVID-19 and imaging confirmation of cerebral infarction or intracranial hemorrhage. During the pandemic peak, only patients with severe COVID-19 complications were hospitalized, and patients with less severe symptoms related to COVID-19 were treated at home. Although hospitals in our system did not mandate the use of specific hard criteria, in general, oxygen saturation $<90 \%$ or $92 \%$ or other signs of severe respiratory distress or severe systemic illness such as sepsis were used as guiding criteria for COVID-19 admission during the crisis. Laboratory data included complete blood count and coagulation profile at hospital admission or in-hospital stroke onset, lowest lymphocyte count, signs of noncerebral end-organ damage (elevated creatinine level $[>1.3 \mathrm{mg} / \mathrm{dL}]$, twice the normal alanine aminotransferase level [>90 U/L] and lactate dehydrogenase level [>484 U/L]), and the highest hospitalization inflammatory (C-reactive protein and ferritin) and coagulopathy markers (D-dimer). Diagnosis of coexistent deep vein thrombosis was recorded. Neuroimaging findings were based on brain CT or MR imaging findings, including stroke

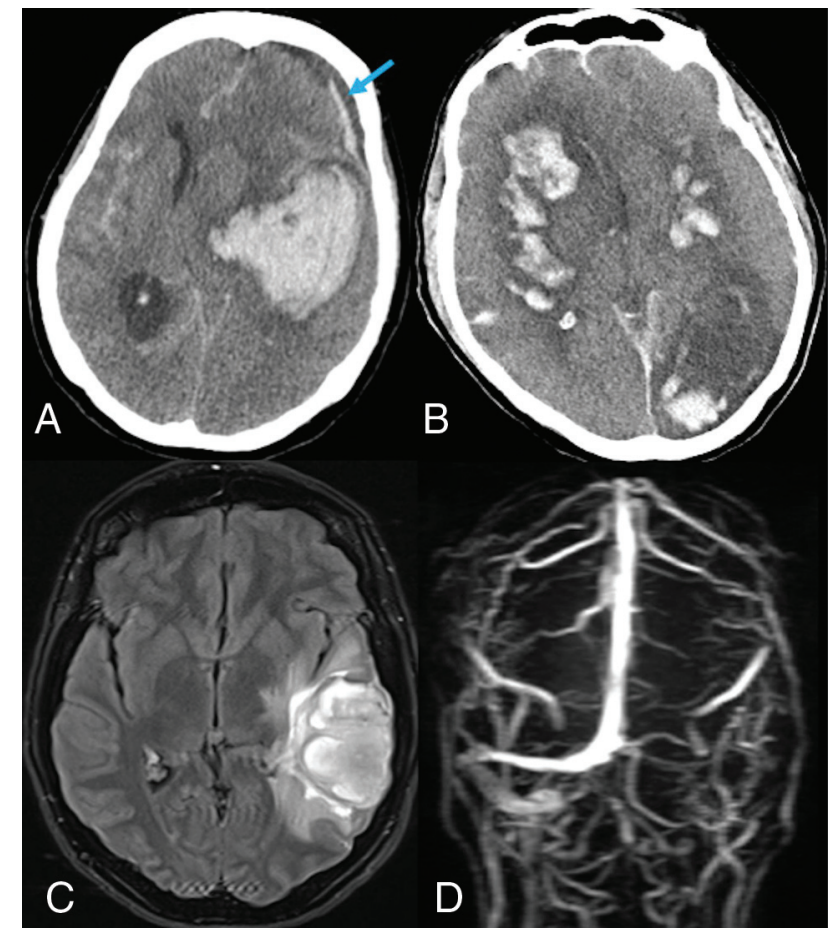

FIG 1. Cerebral hemorrhage in patients with COVID-19. A, Noncontrast CT of the head shows a large left basal ganglia intraparenchymal hemorrhage, with diffuse subarachnoid and left frontal subdural (blue arrow) hemorrhages in a woman with asymptomatic COVID-19. B, Noncontrast CT of the head showing extensive bilateral multifocal cerebral infarctions with hemorrhagic conversion in a comatose man with multiorgan failure. $C$, Brain MR imaging FLAIR and $D$, MR venogram show hemorrhagic venous infarction in the left temporal lobe $(C)$ secondary to thrombosis of left transverse and sigmoid sinuses and internal jugular vein $(D)$ in a young woman presenting with seizures.

subtype, infarction pattern, and the presence of hemorrhagic transformation and simultaneous infarction and hemorrhage.

\section{Statistical Analysis}

A bivariate analysis of imaging and laboratory findings dichotomized by inpatient or outpatient stroke-onset location as a surrogate for COVID-19 severity was performed. Only laboratory variables with at least $75 \%$ complete data were included in our study. Statistical analyses were performed using the Wilcoxon rank sum test for continuous variables and the $\chi^{2}$ test for most categoric variables. The Fisher exact test was used when the cell number was $<5$. Statistical significance was considered for $P$ values $<.05$. All statistical analyses were performed in SAS Version, 9.4 (SAS Institute).

\section{RESULTS}

Between March 14 and April 26, 2020, eighty-six patients (48 men, 38 women) with mean age of 67.4 years (range, 25-94 years) met the inclusion criteria with imaging-confirmed infarction $(83.7 \%)$ or pure intracranial hemorrhage (16.3\%) (Fig 1A). Associated intracranial hemorrhage (Fig $1 B$ ) was found in $20.8 \%$ of 72 patients with infarction, including 9 with simultaneous hemorrhage and infarction and 6 with hemorrhagic transformation, including 1 hemorrhagic venous infarction secondary to 


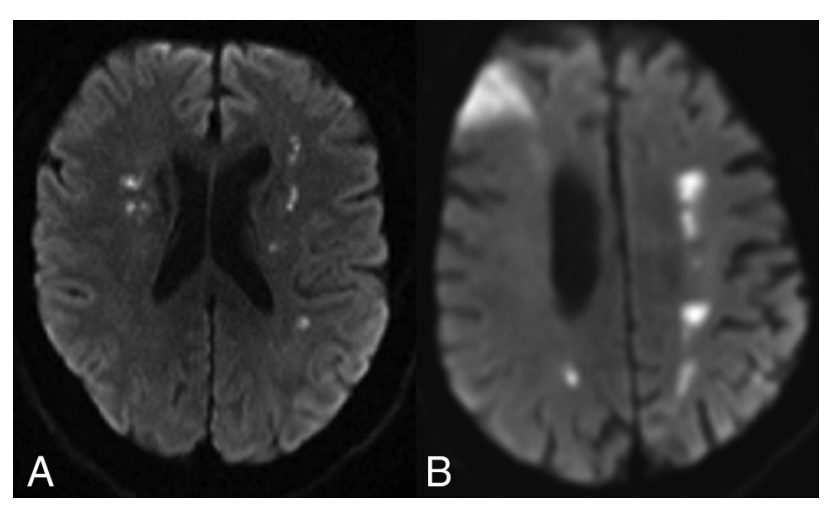

FIG 2. Multivascular territory infarctions in COVID-19. MR imaging of the brain diffusion-weighted imaging demonstrates watershed-pattern infarctions in a 52-year-old man with mild COVID-19 symptoms, who awoke at home with left hemiparesis $(A)$ and an 86-year-old woman admitted for hypoglycemia, dehydration, and COVID-19 pneumonia $(B)$ and found to have new-onset atrial fibrillation; 8 days into her hospitalization, she developed lethargy, left hemiparesis, expressive aphasia, and dysphagia.

dural venous sinus thrombosis (Fig $1 C,-D)$. Multivascular territory infarction (33/72 patients; $45.8 \%$ ) predominated, including 12 with a watershed pattern (Fig 2), followed by single vascular territory infarction (29/72 patients, $40.3 \%)$ and solitary small-vessel occlusion (10/72 patients, $13.9 \%)$.

Forty-one patients (47.7\%) were already hospitalized at stroke onset, whereas 45 patients (52.3\%) developed out-of-hospital neurologic deficits, with ongoing mild or asymptomatic COVID19 infection. In-hospital stroke onset was associated with more frequent mechanical ventilation (56.1\% versus $33.3 \%, P=.034)$, admission tachypnea (respiratory rate, $>20$ breaths per minute; $61.0 \%$ versus $33.3 \%, P=.016$ ), and discharge to a rehabilitation facility (51.2\% versus $22.2 \%, P=.011$ ), supporting our hypothesis that these patients had more severe COVID-19 infection.

Supplemental Table 1 summarizes the bivariate analysis of imaging and laboratory findings dichotomized by patient location at stroke onset (see Supplemental Table 2 for analysis of laboratory values as continuous variables). In-hospital stroke onset was significantly associated with ischemic stroke $(95.1 \%$ versus $73.3 \%$, $P=.006)$, particularly multivascular distribution (56.4\% versus $33.3 \%, P=.022)$ and associated hemorrhagic transformation or simultaneous intracranial hemorrhage ( $31.7 \%$ versus $4.4 \%, P=.001$ ). These patients also had more frequent deep vein thrombosis (29.3\% versus $2.2 \%, P=.001$ ). For those with laboratory values available, patients with in-hospital stroke onset had more frequent leukocytosis $(61.0 \%$ versus $26.7 \%, P=.001)$ and lymphopenia (90.2\% versus 55.6\%, $P=.001$ ), abnormal creatinine levels $(79.0 \%$ versus $43.8 \%, P=.001)$, and severely elevated $\mathrm{D}$-dimer $(92.5 \%$ versus $54.8 \%, P=.001)$, C-reactive protein $(79.0 \%$ versus $43.8 \%$, $P=.002)$, ferritin $(79.0 \%$ versus $31.1 \%, P<.001)$, lactate dehydrogenase $(73.0 \%$ versus $42.9 \%, P=.014)$, and alanine aminotransferase levels (78.1\% versus $39.5 \%, P<.001)$.

\section{DISCUSSION}

Patients hospitalized for severe COVID-19 infection who develop stroke in the hospital demonstrate substantial differences compared with other patients with COVID-19 infection who develop stroke as an outpatient with milder or no COVID-19 symptoms. Although we are unable, in a retrospective study, to prove that our findings are exclusively related to COVID-19, as opposed to other intensive care unit-related variables, patients hospitalized with severe COVID-19 had distinct stroke imaging patterns and even more abnormal laboratory biomarkers, which support a potential pathophysiologic link underpinning our previous finding that COVID-19 is a strong independent risk factor for stroke in hospitalized patients. ${ }^{12}$ Patients with severe manifestations of COVID-19 had significantly more frequent ischemic strokes with multivascular territory distributions, hemorrhagic transformation, and simultaneous infarction and intracranial hemorrhage. While many patients with COVID-19 and stroke had elevated serum inflammatory markers (C-reactive protein and ferritin), lymphocytopenia, thrombocytopenia, and coagulopathy (elevated D-dimer levels and international normalized ratio), those with severe consequences of COVID-19 had significantly higher serum markers of inflammation, with more frequent levels of ferritin $>4$ times normal and Creactive protein levels more than twice normal. These patients also had significantly higher markers of hypercoagulability with Ddimer levels more frequently $>4$ times normal. Furthermore, patients with severe COVID-19 and stroke had significant associations with greater frequencies of leukocytosis, lymphopenia, and cytolysis (measured by lactate dehydrogenase), hepatic and renal dysfunction, and deep vein thrombosis.

In a series of 214 patients hospitalized with COVID-19 from Wuhan, China, acute cerebrovascular disease was first reported in 6 patients $(2.8 \%)$ and was associated with severe pulmonary infection, older age, stroke risk factors, elevated inflammatory markers, and end-organ damage. ${ }^{8}$ Evidence of a concomitant prothrombotic state was proposed by others who describe a series of 6 patients with COVID-19 and ischemic stroke who had infarctions suggesting large-artery occlusion. ${ }^{9}$ In that study, all cases had significantly elevated D-dimer levels, 2 had venous thromboembolic disease, and 2 developed large-artery occlusion despite therapeutic anticoagulation. One patient had antiphospholipid antibodies. Very high median D-dimer and C-reactive protein levels were also reported in a larger retrospective study of 32 patients with COVID-19 with ischemic stroke in the New York metropolitan area. ${ }^{10}$ In a study by others in the same region of 31 patients with COVID-19 with ischemic stroke, higher D-dimer levels and erythrocyte sedimentation rates were reported compared with concurrent patients with COVID-19 without stroke and with a historical sample of patients with influenza. ${ }^{13}$ In that study, when one adjusted for demographics, vascular risk factors, and critical care admission, COVID-19 still nearly quintupled the odds of ischemic stroke compared with influenza infection.

Our experience, like that of other centers, ${ }^{9-11,13,14}$ suggests that a hypercoagulable-prothrombotic state is a prevalent homeostatic complication among patients with COVID-19 and particularly in those with severe illness. Under these conditions, in which sepsis is common, ineffective host defense mechanisms to limit crosstalk between coagulation and hyperinflammation can increase tissue injury, worsen organ dysfunction, and promote thrombogenesis. ${ }^{15,16}$ Hyperinflammation can activate coagulation pathways via several mechanisms. These include promoting 
Supplemental Table 1: Bivariate analysis of imaging and laboratory findings in patients with COVID-19 dichotomized by location at stroke onset

\begin{tabular}{|c|c|c|c|c|}
\hline Variable & Median (IQR) & In-Hospital (No.) (\%) & Out-of-Hospital (No.) (\%) & $P$ Value \\
\hline Patients (No.) & 86 & 41 & 45 & \\
\hline \multicolumn{5}{|l|}{ Age (yr) } \\
\hline $20-69$ years of age & $68(60-76)$ & $20(48.8)$ & $26(57.8)$ & .403 \\
\hline Sex, male & & $26(63.4)$ & $22(48.9)$ & .176 \\
\hline \multicolumn{5}{|l|}{ Imaging } \\
\hline Ischemic stroke & & 39 (95.1) & $33(73.3)$ & .006 \\
\hline Infarction, no hemorrhage & & $26(63.4)$ & $31(68.9)$ & .001 \\
\hline Infarction with HT & & $5(12.2)$ & $1(2.2)$ & \\
\hline Infarction and ICH & & $8(19.5)$ & $1(2.2)$ & \\
\hline Pure $\mathrm{ICH}$, no infarction & & $2(4.9)$ & $12(26.8)$ & \\
\hline Multivascular territory infarction $(n=72)$ & & $22(56.4)$ & $11(33.3)$ & .022 \\
\hline Deep vein thrombosis & & $12(29.3)$ & $1(2.2)$ & .001 \\
\hline \multicolumn{5}{|l|}{ Laboratory values } \\
\hline \multicolumn{5}{|l|}{ White blood cell ${ }^{\mathrm{a}}$} \\
\hline$\geq 10.5 \mathrm{~K} / \mathrm{uL}$ & $9.7(7.2-14.7)$ & $25(61.0)$ & $12(26.7)$ & .001 \\
\hline \multicolumn{5}{|l|}{ Lymphocytes ${ }^{b}$} \\
\hline$<1.00 \mathrm{~K} / \mathrm{uL}$ & $0.6(0.31-1.05)$ & $37(90.2)$ & $25(55.6)$ & .001 \\
\hline \multicolumn{5}{|l|}{ Hemoglobin $^{a}$} \\
\hline$<11.5 \mathrm{~g} / \mathrm{dL}$ & $12.3(9.7-13.8)$ & $19(46.3)$ & $16(35.6)$ & .309 \\
\hline \multicolumn{5}{|l|}{ Platelets $^{\mathrm{a}}$} \\
\hline $\begin{array}{l}<150 \mathrm{~K} / \mathrm{uL} \\
\mathrm{aPTT}{ }^{\mathrm{a}, \mathrm{c}}\end{array}$ & \multicolumn{3}{|c|}{$\mathrm{aPTT}{ }^{\mathrm{a}, \mathrm{c}}$} & .976 \\
\hline$>36.3$ seconds & $30.9(28.4-36.1)$ & $13(32.5)$ & $7(17.1)$ & .108 \\
\hline \multicolumn{5}{|l|}{$I N R^{a, c}$} \\
\hline$>1.20$ & $1.17(1.07-1.31)$ & $19(48.7)$ & $17(39.5)$ & .403 \\
\hline \multicolumn{5}{|l|}{ D-dimer, ${ }^{\mathrm{c}} 4 \times \mathrm{nl}$} \\
\hline$>920 \mathrm{ng} / \mathrm{mL}$ & 3060 (1064-24,211) & $37(92.5)$ & $17(54.8)$ & .001 \\
\hline $\begin{array}{l}\text { C-reactive protein, }{ }^{\mathrm{C}} 2 \times \mathrm{nl} \\
>10.0 \mathrm{mg} / \mathrm{L}\end{array}$ & $17.5(6.4-28.8)$ & $30(79.0)$ & $14(43.8)$ & .002 \\
\hline \multicolumn{5}{|l|}{ Ferritin, ${ }^{c} 4 \times \mathrm{nl}$} \\
\hline$>1200 \mathrm{ng} / \mathrm{mL}$ & 1382 (706-2424) & $30(79.0)$ & $10(31.1)$ & $<.001$ \\
\hline \multicolumn{5}{|l|}{$\mathrm{LDH}^{\mathrm{c}}, 2 \times \mathrm{nl}$} \\
\hline$>484 \mathrm{U} / \mathrm{L}$ & $536(357-815)$ & $27(73.0)$ & $12(42.9)$ & .014 \\
\hline \multicolumn{5}{|l|}{$\mathrm{ALT}^{\mathrm{c}}, 2 \times \mathrm{nl}$} \\
\hline$>90.0 \mathrm{U} / \mathrm{L}$ & $50(29-136)$ & $32(78.1)$ & $17(39.5)$ & $<.001$ \\
\hline \multicolumn{5}{|l|}{ Creatinine $^{c}$} \\
\hline$>1.30 \mathrm{mg} / \mathrm{dL}$ & $1.30(0.93-2.81)$ & $28(70.0)$ & $15(34.1)$ & .001 \\
\hline
\end{tabular}

Note:-HT indicates hemorrhagic transformation; ICH, intracranial hemorrhage; nl, upper limit of the normal value; aPTT, activated partial thromboplastin time; INR, international normalized ratio; LDH, lactate dehydrogenase; ALT, alanine aminotransferase; IQR, interquartile range.

a White blood cell count, hemoglobin, platelets, activated aPTT, and INR are from admission or at the time of consultation for stroke. Remaining laboratory values (except lymphocyte count) are the maximum level during the hospitalization.

${ }^{\mathrm{b}}$ Lowest lymphocyte count during admission.

${ }^{c}$ Variable has missing values. The number and percentage (No.) (\%) of patients with missing laboratory values are the following: aPTT ( $\left.n=5,5.8 \%\right)$, INR $(n=4,4.7 \%)$, Ddimer $(n=16,18.6 \%)$, C-reactive protein $(n=16,18.6 \%)$, ferritin $(n=16,18.6 \%)$, LDH $(n=21,24.4 \%)$, ALT, $(n=2,2.3 \%)$, and creatinine $(n=2,2.3 \%)$. All other variables have no missing values.

endothelial activation, increasing tissue factor expression on vascular endothelial and circulating inflammatory cells such as monocytes, and downregulating the efficiency of the protein $\mathrm{C}$-protein $\mathrm{S}$ thrombomodulin system to regulate the increased thrombogenesis associated with consumptive coagulation. ${ }^{14,17,18}$ Specifically, surges in proinflammatory cytokines and mediators, related to an exaggerated innate immune response and extensive tissue damage associated with severe COVID-19, can downregulate the expression and function of thrombomodulin, an endogenous protein with potent anticoagulant and anti-inflammatory properties pivotal in preventing vascular occlusion. ${ }^{19,20}$

Moreover, an autoimmune hypercoagulable state associated with antiphospholipid antibodies, such as anti-cardiolipin and anti- $\beta_{2}$-glycoprotein-1 antibodies, can contribute to the pathophysiology of a subset of strokes associated with COVID-19. ${ }^{21}$ As shown in the peripheral vasculature, ${ }^{22}$ direct SARS-CoV-2 infection of cerebrovascular endothelia expressing angiotensinconverting enzyme 2, causing endotheliitis and vascular injury, is postulated and may result in both ischemic and hemorrhagic stroke, though this mechanism remains unproven. Finally, a potential downregulation of neurovascular endothelial angiotensin-converting enzyme 2 expression in response to its binding to SARS-CoV-2 may also be relevant to ischemic stroke pathogenesis, though this too is theoretic.

Our study has several strengths and limitations. As we previously reported, ${ }^{12}$ our series likely undercounted the actual COVID-19-stroke burden during this timeframe. Many potential patients with stroke with normal initial brain imaging did not undergo repeat imaging because of clinical instability or concerns for spreading infection while transporting patients with COVID19 off quarantined wards. For similar reasons, MR imaging use was limited at numerous centers, especially earlier on in the local 
epidemic. Therefore, some patients with smaller and more acute infarctions were not included in our study, and our results should be interpreted as only relevant to patients with COVID-19 who have imaging-confirmed stroke.

A relative strength to our approach was the inclusion of patients with primary and secondary intracranial hemorrhage, allowing, for the first time, the demonstration of a frequent hemorrhagic component to infarctions seen in patients with severe COVID-19. Independent neuroradiologic review of the imagingenabled confirmation of stroke diagnoses and characterization of imaging findings were blinded and uniform. As a retrospective study, some laboratory values were missing in a proportion of patients. To mitigate this issue, we analyzed only variables with at least $75 \%$ complete data. Consequently, some commonly cited laboratory abnormalities associated with COVID-19 are not included, such as fibrinogen, antiphospholipid antibodies, and interleukin levels. In addition, given our small sample size, we were unable to perform an adjusted analysis. Nevertheless, the number of cases of COVID-19 stroke in our series permitted a comprehensive analysis of neuroimaging and laboratory biomarkers, reinforcing potential pathophysiologic mechanisms supporting the association between COVID-19 and stroke.

\section{CONCLUSIONS}

Compared with those with mild or asymptomatic COVID-19 at stroke onset, patients who have strokes while hospitalized with severe manifestations of COVID-19 have significantly more inflammation, multiorgan dysfunction, and coagulopathy, including deep vein thrombosis. These findings support proposed pathophysiologic mechanisms linking these diagnoses, though we cannot definitively state that our findings are specific to SARS-CoV-2 infection as opposed to the critical illness associated with severe COVID-19. Infarctions with concurrent hemorrhage are also more frequent. Given the associated hypercoagulability, clinical-treatment algorithms for patients hospitalized with COVID-19 have shifted toward widespread use of full- or prophylactic-dose anticoagulation to prevent thromboembolic complications, though the benefits of this approach remain unproven because it is based solely on observational data. ${ }^{23}$ In patients with COVID-19 with stroke, anticoagulation may be of benefit, but the approach may have to be more nuanced, given the risks of associated cerebral hemorrhage, mandating a more cautious balancing of the risks of thrombosis and hemorrhage.

Disclosures: Christopher G. Filippi-UNRELATED: Consultancy: Guerbet, Syntactx; Grants/Grants Pending: Foundation of the American Society of Neuroradiology grant and National MS Society grant*; Payment for Manuscript Preparation: article on topics in MRI journal (April 2020); Stock/Stock Options: minority stakeholder in start-up Avicenna. Pina Sanelli-UNRELATED: Grants/Grants Pending: Siemens, Harvey L. Neiman Sanell Health Policy Institute, Comments: research partnership.* Souhel Najjar-UNRELATED: Employment: Full-time and Chair of Neurology at Northwell Health. *Money paid to the institution.

\section{REFERENCES}

1. Zhu N, Zhang D, Wang W, et al; China Novel Coronavirus Investigating and Research Team. A novel coronavirus from patients with pneumonia in China, 2019. N Engl J Med 2020;382:727-33 CrossRef Medline
2. COVID-19 dashboard by the Center for Systems Science and Engineering at Johns Hopkins University. https://publichealthupdate. com/jhu/. Accessed October 1, 2020

3. Mizumoto K, Kagaya K, Zarebski A, et al. Estimating the asymptomatic proportion of coronavirus disease 2019 (COVID-19) cases on board the Diamond Princess cruise ship, Yokohama, Japan, 2020. Euro Surveill 2020;25:2000180 CrossRef Medline

4. Richardson S, Hirsch JS, Narasimhan M, et al. Presenting characteristics, comorbidities and outcomes among 5700 patients hospitalized with COVID-19 in the New York City area. JAMA 2020;323:2052-59 CrossRef Medline

5. Mehta P, McAuley DF, Brown M, et al; HLH Across Speciality Collaboration, UK. COVID-19: consider cytokine storm syndromes and immunosuppression. Lancet 2020;395:1033-34 CrossRef Medline

6. Tang N, Li D, Wang X, et al. Abnormal coagulation parameters are associated with poor prognosis in patients with novel coronavirus pneumonia. J Thromb Haemost 2020;18:844-47 CrossRef Medline

7. Cao W, Li T. COVID-19: towards understanding of pathogenesis. Cell Res 2020;30:367-69 CrossRef Medline

8. Mao L, Jin H, Wang M, et al. Neurologic manifestations of hospitalized patients with coronavirus disease 2019 in Wuhan, China. JAMA Neurol 2020;77:683 CrossRef Medline

9. Beyrouti R, Adams ME, Benjamin L, et al. Characteristics of ischaemic stroke associated with COVID-19. J Neurol Neurosurg Psychiatry 2020;91:889-91 CrossRef Medline

10. Yaghi S, Ishida K, Torres J, et al. SARS2-CoV-2 and stroke in a New York healthcare system. Stroke 2020;51:2002-11 CrossRef Medline

11. Arachchillage DR, Laffan M. Abnormal coagulation parameters are associated with poor prognosis in patients with novel coronavirus pneumonia. J Thromb Haemost 2020;18:1233-34 CrossRef Medline

12. Katz JM, Libman RB, Wang JJ, et al. Cerebrovascular complications of COVID-19. Stroke 2020;51:e227-31 CrossRef Medline

13. Merkler AE, Parikh NS, Mir S, et al. Risk of ischemic stroke in patients with coronavirus 2019 (COVID-19) vs patients with influenza. JAMA Neurol 2020 Jul 2. [Epub ahead of print] CrossRef Medline

14. Lillicrap D. Disseminated intravascular coagulation in patients with 2019-nCoV pneumonia. J Thromb Haemost 2020;18:786-87 CrossRef Medline

15. Foley JH, Conway EM. Cross talk pathways between coagulation and inflammation. Circ Res 2016;118:1392-1408 CrossRef Medline

16. Levi M, van der Poll T. Coagulation and sepsis. Thromb Res 2017;149:38-44 CrossRef Medline

17. Amiral J, Seghatchian J. Revisiting the activated protein C-protein S-thrombomodulin ternary pathway: impact of new understanding on its laboratory investigation. Transfus Apher Sci 2019;58:53844 CrossRef Medline

18. Bode M, Mackman N. Regulation of tissue factor gene expression in monocytes and endothelial cells: thromboxane A2 as a new player. Vascul Pharmacol 2014;62:57-62 CrossRef Medline

19. Ikezoe T. Thrombomodulin/activated protein $\mathbf{C}$ system in septic disseminated intravascular coagulation. J Intensive Care 2015;3:1 CrossRef Medline

20. Joseph L, Fink LM, Hauer-Jensen M. Cytokines in coagulation and thrombosis: a preclinical and clinical review. Blood Coagul Fibrinolysis 2002;13:10-16 CrossRef Medline

21. Zhang Y, Xiao M, Zhang S, et al. Coagulopathy and antiphospholipid antibodies in patients with COVID-19. N Engl J Med 2020;382: e38 CrossRef Medline

22. Varga Z, Flammer AJ, Steiger P, et al. Endothelial cell infection and endotheliitis in COVID-19. Lancet 2020;395:1417-18 CrossRef Medline

23. Nadkarni GN, Lala A, Bagiella E, et al. Anticoagulation, mortality, bleeding and pathology among patients hospitalized with COVID19: a single health system study. J Am Coll Cardiol 2020 Aug 24. [Epub ahead of print] CrossRef Medline 